\title{
Reality versus Models: What We Learned From Acoustic Control Experiments
}

\author{
Clark J Radcliffe \\ Professor of Mechanical Engineering \\ Michigan State University \\ East Lansing, MI 48824 \\ email: radcliffe@me.msu.edu
}

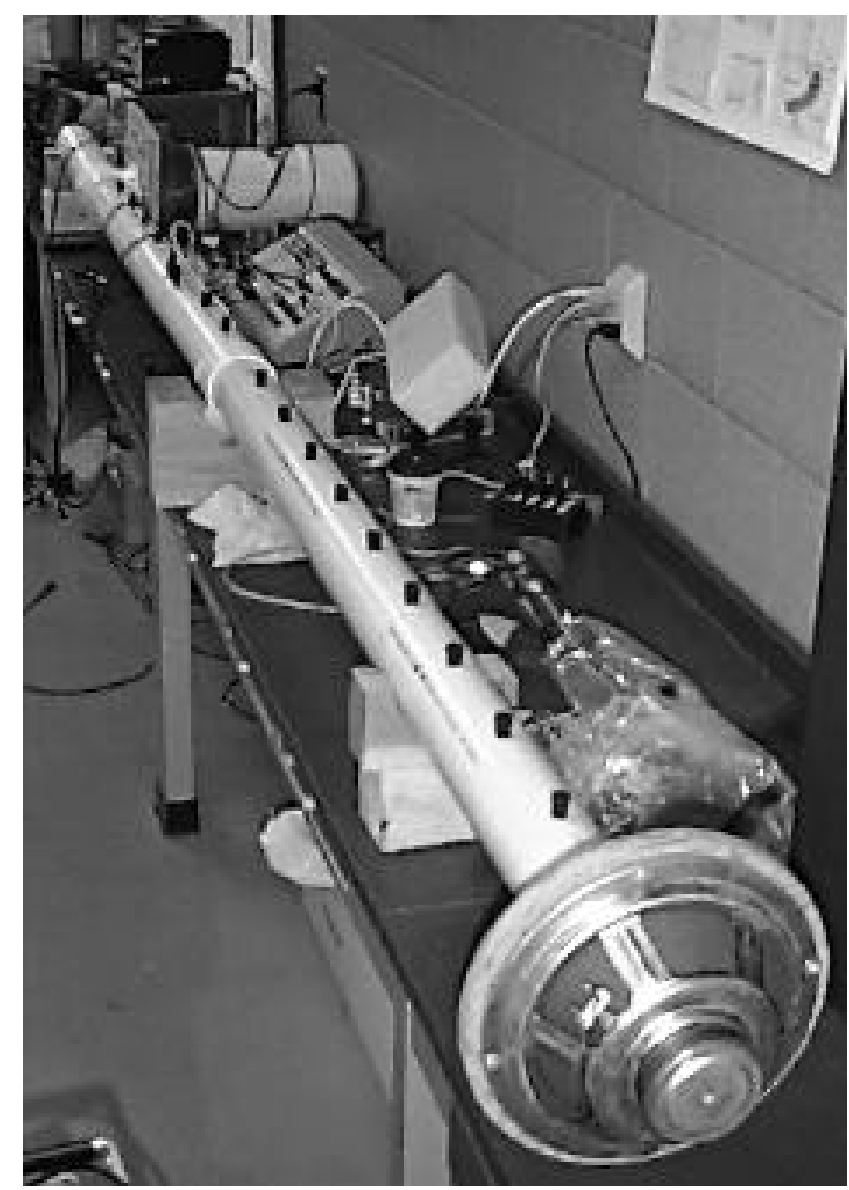

Figure 1: The Acoustic Duct Test Stand. Shown are the Excitation speaker in the foreground, microphone test locations along the duct and the control speaker near the far end

\section{Introduction}

The development of active acoustic control experiments demonstrates the need to calibrate our view of the physical world with regular testing. We started with a desire to use feedback-based active control on acoustic systems with a system model based on the wave equation and the opinion that all work would flow smoothly from that model of physical reality. We have now realized that the hardest issues associated with active acoustic control are not well described by our simplistic initial model. Physical testing is the vehicle though which we tested our initial model and found it lacking. With physical testing and experimentation, we have focused on those elements of acoustics that are most important to the success of our acoustic control methodologies.

\section{The 1-D Acoustics Problem}

Our evolution started with the simple 1 dimensional wave equation

$$
\begin{aligned}
\frac{\partial^{2} \mathrm{u}(\mathrm{x}, \mathrm{t})}{\partial \mathrm{t}^{2}}-\mathrm{c}^{2} \frac{\partial^{2} \mathrm{u}(\mathrm{x}, \mathrm{t})}{\partial \mathrm{x}^{2}} & \\
=-\frac{\partial}{\partial x}\left[\frac{\delta(x) P(t)}{\rho}\right] & \\
& -\sum_{i=1}^{k}\left[\delta\left(x-x_{i}\right)\right] \frac{\partial}{\partial t}\left[\frac{M_{i}(t)}{\rho S}\right]
\end{aligned}
$$

where $\mathrm{K}=$ complex impedance of the termination end (dimensionless). When $\operatorname{Re}(\mathrm{K})$ equals zero or is infinity, the termination end of the duct reflects all the acoustic energy and the response is composed of standing waves only. All other values of K yield 


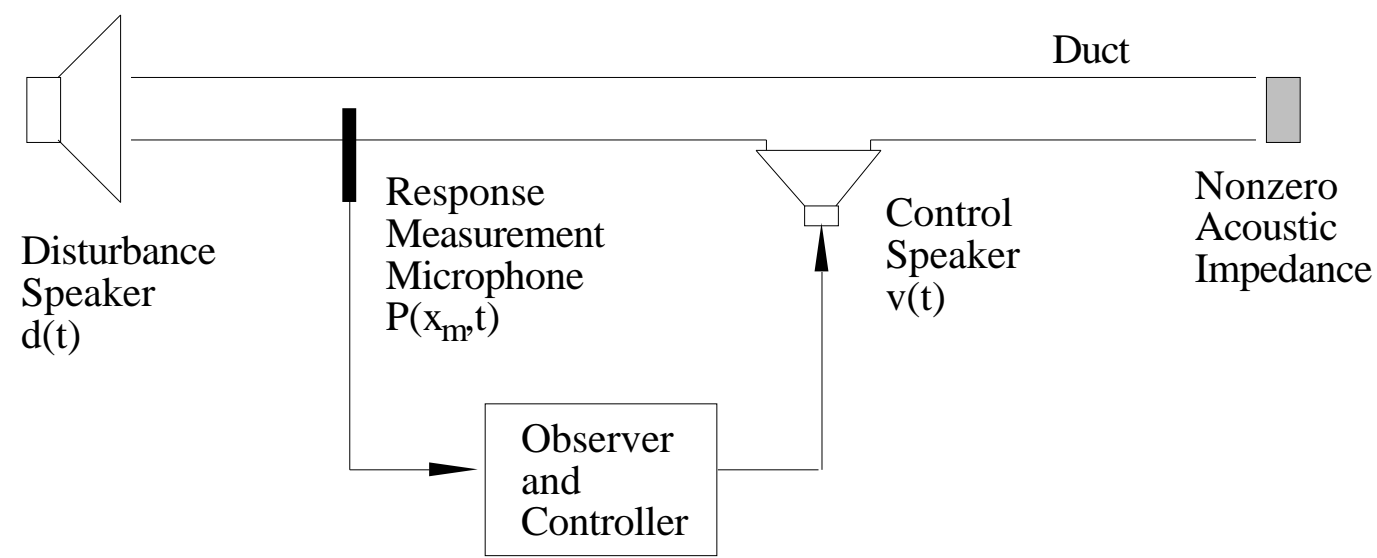

Figure 2. Active Noise Control System

some combination of propagating and standing wave response (Spiekermann and Radcliffe, 1988a). When $K=1+0 \mathrm{i}$ the termination end of the duct absorbs all the acoustic energy and the response is composed of propagating waves only. In general, the reflection coefficient gives the relative magnitude of the reflected pressure wave. The real part of $\mathrm{K}$ (acoustic resistance) is associated with energy dissipation. The imaginary part of $\mathrm{K}$ (acoustic reactance) is associated with conservative fluid compliance and/or inertia effects.

$$
\begin{aligned}
\frac{\partial u}{\partial x}(L, t)=-K\left(\frac{1}{c}\right) & \frac{\partial u}{\partial t}(L, t) \\
& K \neq 0+0 i, 1+0 i, \infty
\end{aligned}
$$

The duct end at $\mathrm{x}=0$ is modeled as a totally reflective, open end. This boundary condition is

$$
\frac{\partial u}{\partial x}(0, t)=0
$$

The acoustic pressure of the system is related to the spatial gradient of the particle displacement by (Seto, 1973)

$$
P(x, t)=-\rho c^{2} \frac{\partial u}{\partial x}(x, t)
$$
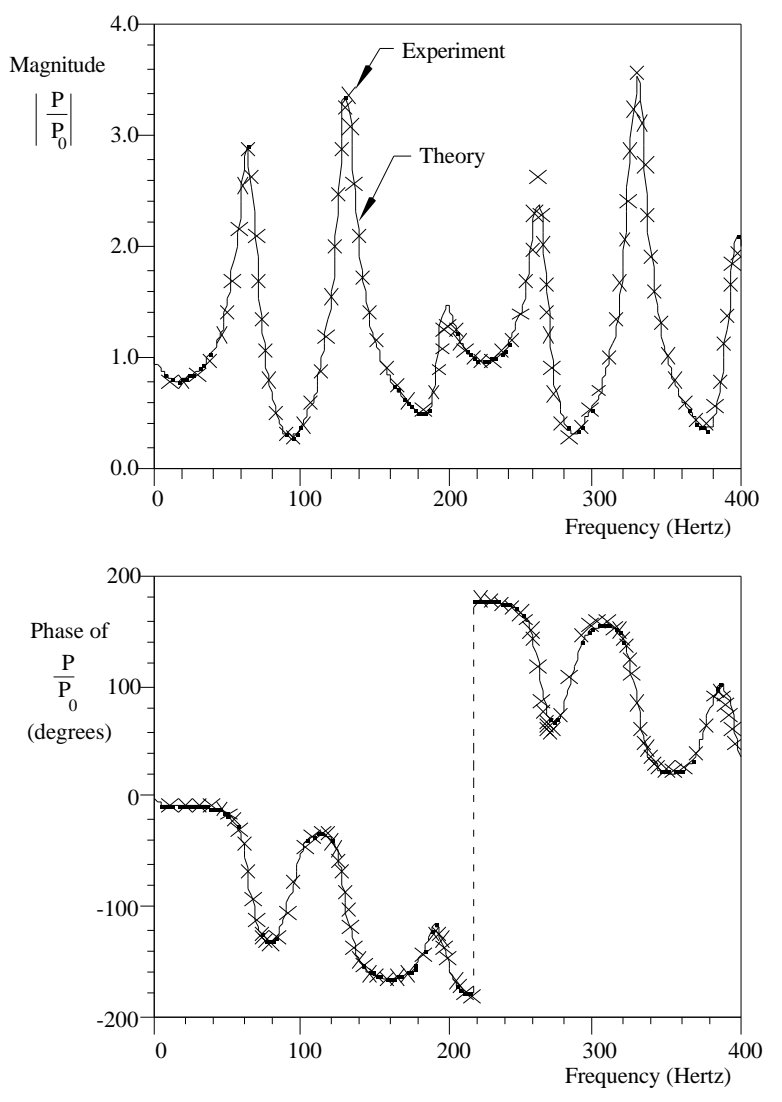

Figure 3: Acoustic Duct Pressure Response Verification. Wave equation predicted response shown as solid line. Measured responses shown with "X" symbols. Duct model parameters: length, $\mathrm{L}=2.6 \mathrm{~m}$ and Impedance, $\mathrm{K}=0.285+0.079 \mathrm{I}$ (measured) 


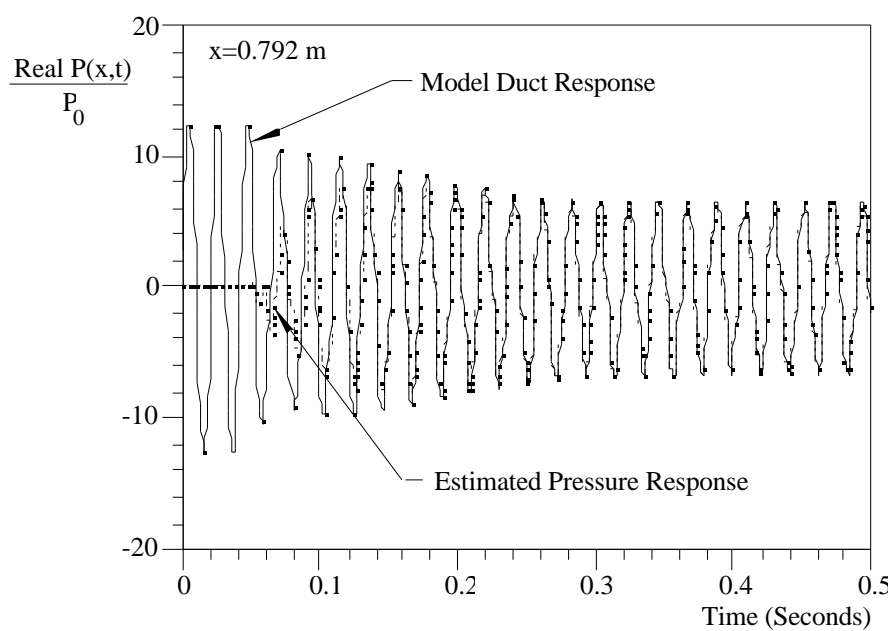

Figure 4: State Observer Transient Response Verification. All values are measured. "Model" refers to Acoustic duct test stand model microphone measurements. "Estimated" refers to real-time predictions from the state-space observer implementation. Note the rapid speed of convergence.

\section{The 1-D Duct Experiment}

An acoustic duct test stand was designed and constructed (A.J. Hull and C. J. Radcliffe, 1992 ) (Fig. 1). The test stand was designed so that its responses would follow the above mathematical model as closely as possible. The duct test stand is a segment of a larger control system test stand (Fig. 2). The wave equation model predicted the physical measurements of acoustic responses quite well (Fig. 3).

Based on the model success, a state-space observer was developed and implemented on a DSP system in real time. The observer tracked the duct response successfully (A.J. Hull, C.J. Radcliffe and C.R. MacCluer, 1991) (Fig. 4). When statespace feedback control was implemented, the measured closed-loop responses demonstrated the ability to control duct dynamics (Fig. 5) within the bandwidth of the audio speaker used as the actuator (A.J. Hull, C.J. Radcliffe, and S.C. Southward, 1993). The actuator dynamics yielded a limited actuator bandwidth (Fig. 6) and were the limiting factor in our ability to implement feedback control.
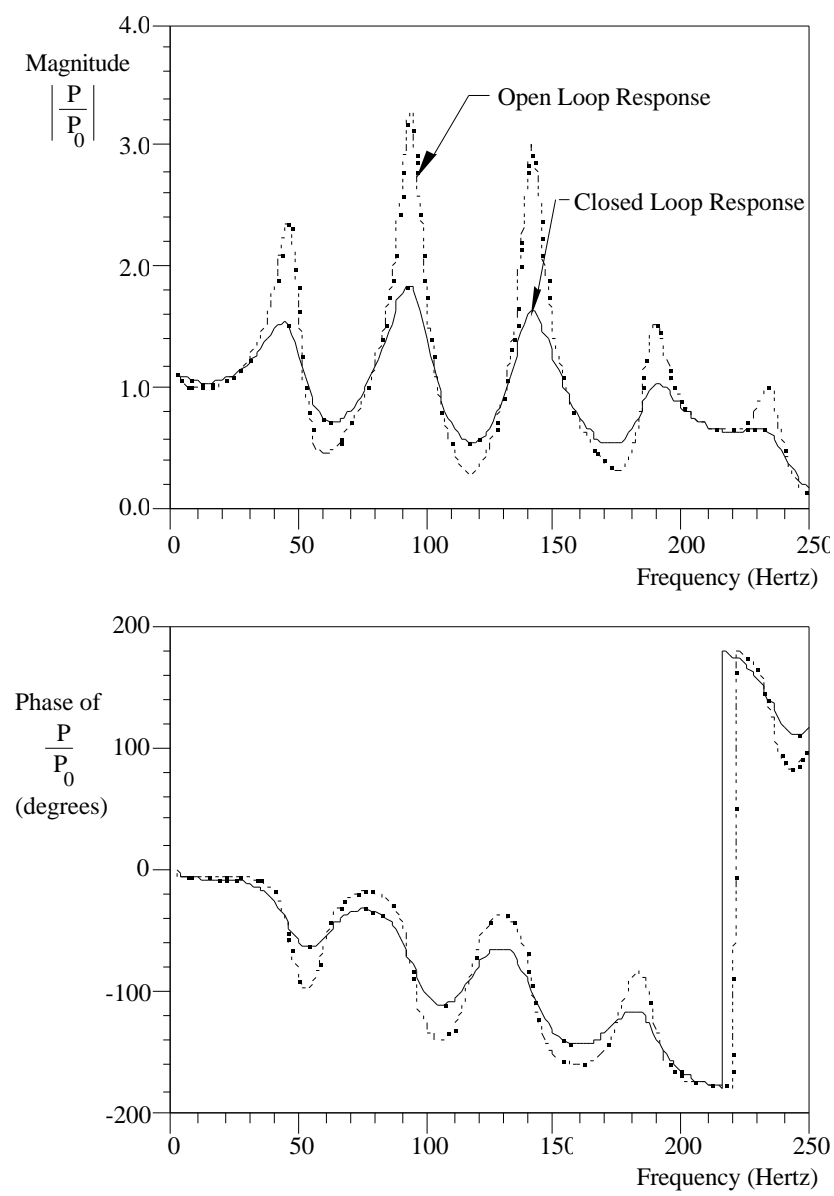

Figure 5: Measured Closed-Loop State Feedback Response of the Acoustic Duct Test Stand Compared with Open-Loop, Uncontrolled Response

\section{What We Learned}

The control experiment caused a change in research direction from control design to actuator design. In fact, the current research has yielded feedback controlled speakers with wide bandwidths (Fig. 7) more suitable to feedback control implementations (Radcliffe, C. J., and Gogate, S. D., 1996). These feedback compensated speakers have capabilities with applications far beyond acoustic control. 

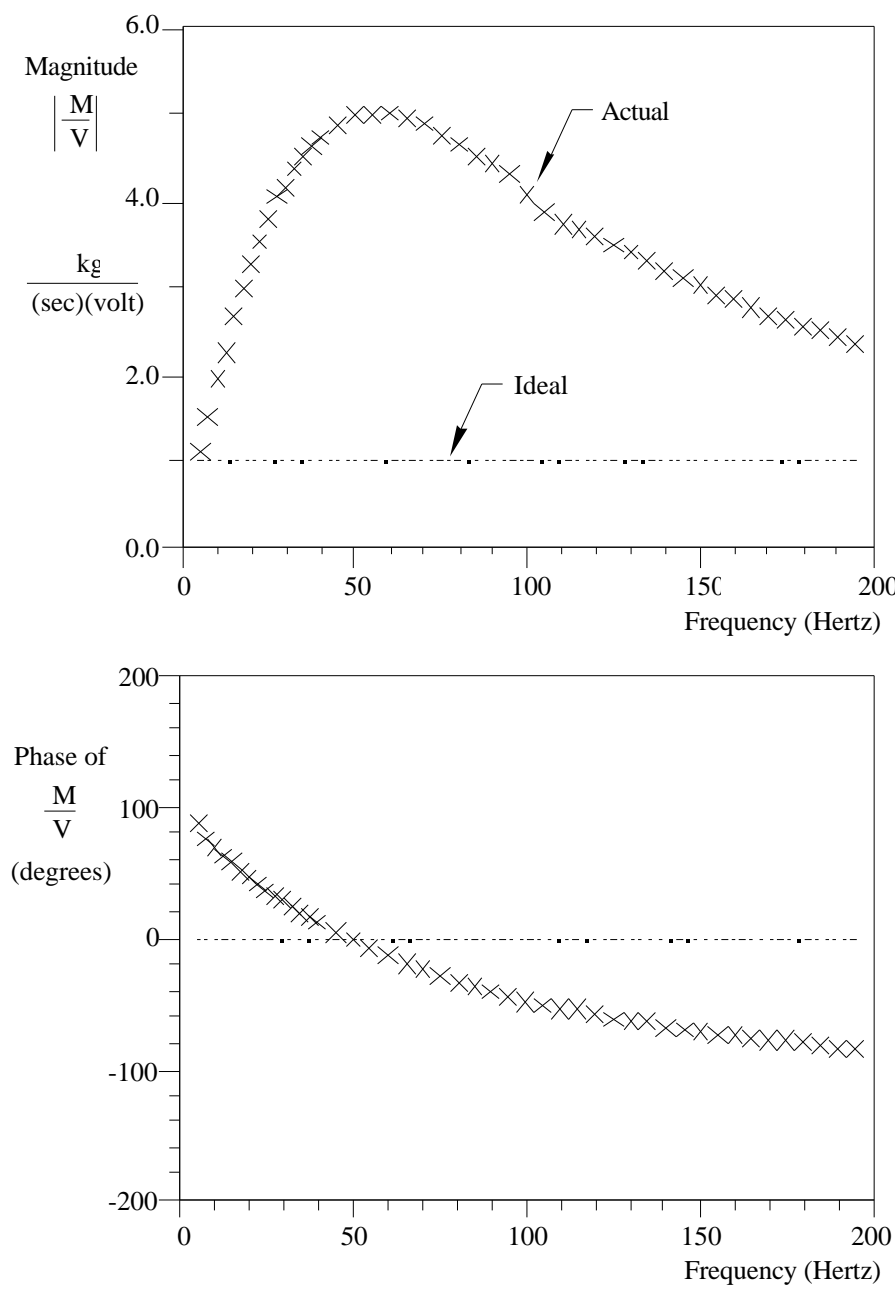

Figure 6: Measured Frequency Response of the Acoustic Speaker used as an actuator in the Acoustic Control Experiment

\section{References:}

Hull, A.J., Radcliffe, C.J., Miklav čč , M., MacCluer, C.R. (1990), "State Space Representation of the Nonself-Adjoint Acoustic Duct System", Journal of Vibration and Acoustics, 112, pp. 483-488.

C. Spiekermann and C.J. Radcliffe, 1988, "Decomposing One-dimensional Acoustic Pressure Response into Propagating and Standing Waves", J. Acoustical Society of America, 84(4), pp. 1536-1541, Oct.

A.J. Hull and C.J. Radcliffe, 1992, "Experimental Verification of the Nonself-Adjoint State Space Duct Model", , ASME Journal of Vibration and Acoustics, Vol. 114, No. 3,

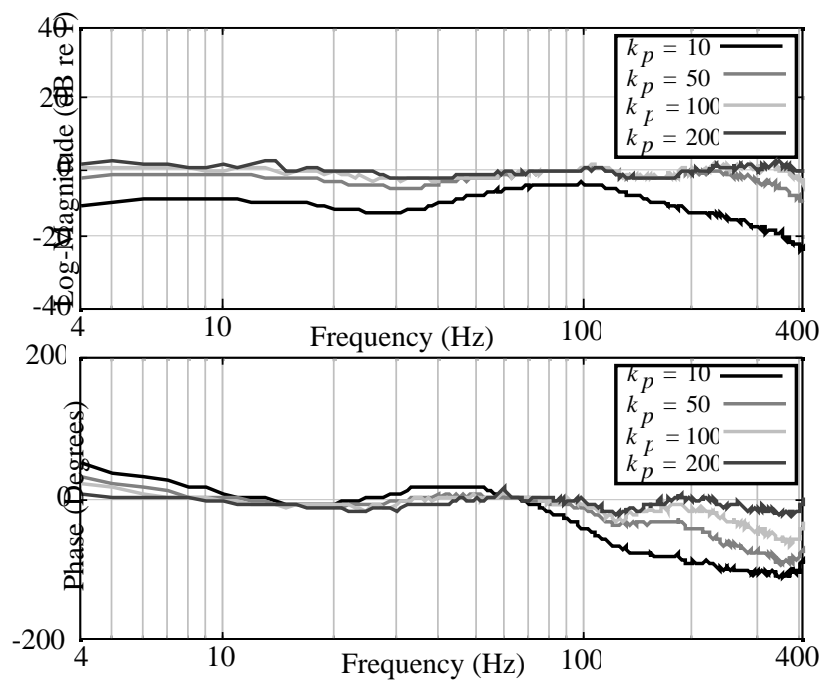

Figure 7: Closed-Loop Feedback Compensated Audio Speaker Response Showing bandwidth increased from approximately $50 \mathrm{~Hz}$ Open-Loop to more than $400 \mathrm{~Hz}$. with Feedback Compensation

pp.404-408, July, and presented 1990 ASME Winter Annual Meeting, Dallas, Texas, 90WA/NCA-5.

A.J. Hull, C.J. Radcliffe and C.R. MacCluer, 1991,"State Estimation of a Nonself-Adjoint Acoustic System", , ASME J. Dynamic Systems, Measurement and Control, Vol. 113, pp. 122-126, Mar.. and presented 1990 ASME Winter Annual Meeting, Dallas, Texas, ASME Bk. No G00550.

A.J. Hull, C.J. Radcliffe, and S.C. Southward, 1993, "Global Active Noise Control of a One Dimensional Acoustic Duct Using a Feedback Controller, ASME J. Dynamic Systems, Measurement and Control, Vol. 115, pp.488494, Sept., and presented 1991 ASME Winter Annual Meeting.

Radcliffe, C.J., and Gogate, S.D., 1996, "Velocity Feedback Compensation Of Electromechanical Speakers For Acoustic Applications ", paper 3a-07-1, Proceedings, 1996 Iinternational Federation Of Automattic Control World Congress, San Francisco, California, USA, June 30-July 5. 